ADDENDUM

https://doi.org/10.1038/s41586-018-0233-9

\title{
Addendum: Freezing on a sphere
}

Rodrigo E. Guerra, Colm P. Kelleher, Andrew D. Hollingsworth \& Paul M. Chaikin

Addendum to: Nature https://doi.org/10.1038/nature25468, published online 14 February 2018.

After the publication of our Letter, we were made aware of some pioneering simulations by Prestipino Giarrita et al. that investigated the icosahedral ordering of defects in assemblies of hard particles packed on the surfaces of spheres ${ }^{1,2}$. This work anticipated the notion that lattice defects were an inevitable part of ordered structures assembled on spherical shells, and they defined a pair defect-density correlation ${ }^{2}$, $g_{\text {def }}$, similar to the $g_{55}$ function that we plot in Fig. 2 a of our Letter. This work $^{1,2}$ represents a solid contribution to the field and merits wider recognition.

1. Prestipino Giarrita, S., Ferrario, M. \& Giaquinta, P. V. Statistical geometry of hard particles on a sphere. Physica A 187, 456-474 (1992).

2. Prestipino Giarrita, S., Ferrario, M. \& Giaquinta, P. V. Statistical geometry of hard particles on a sphere: analysis of defects at high density. Physica A 201, 649-665 (1993). 